\title{
RESISTEN EIA AO IMPACTO DE REVESTIMENTOS PARA FUNDIÇÕES ODONTOLÓGICAS, EM DIVERSAS CONDIÇÕES DE TEMPERATURA NO FORNO
}

\author{
Prof. Léo Werner Süffert * \\ Prof. Fernando Lyra Martins **
}

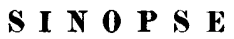

Os investigadores procuram verificar e comparar a resistência ao impacto ou choque, em quatro marcas comerciais de revestimentos 0 lontológicos, ell função da perda de calor verificada nos corpos de prova em diversos espaços de tempo, ao serem retirados do forno à temperatura de $700 \%$. Chama atenção para o relacionamento gráfico deste tipo de experiências, na forma de curvas elaboradas simplesmente à partir de médias de ensaios. Quando não forem corroboradas por uma análise estatística, podem induzir facilmente à deduções e conclusões errôneas.

\section{INTRODUCX̃O}

Nos processos de fundições, o material refratário (revestimento) é levado ao forno, cuja temperatura deverá atingir $700^{\circ} \mathrm{C}$, na maioria das técnicas. Com este aquecimento objetiva-se: $1^{\circ}$ a eliminação da cera que serviu como matriz e que deverá ser volatilizada sem deixar resíduos, e $2^{\circ}$ obter um aumento volu- métrico da massa de revestimento com a finalidade de compensar contrações já ocorridas durante a elaboração da matriz de cera e contrações que irão ocorrer durante a solidificação da liga de ouro.

Através de um canal de «alimentação» e, por forças de centrifugação, pressão de ar ou gases, ou ainda, prir sucção (vacuo), a liga de ouro em estado líquido é jogada para o iı.ter'or da massa de revestimento. oca. sionando nesta última um CHOQLE ou IMPACTO.

De acordo com as condições de temperatura em que se encortra ir massa do rovestimento, não seria de se supor que poderá variar a sua resistência ao impacto?

No decorrer da revisão da bibliografia, encontramos algumas referências sobre o assunto, embora abordassem o mesmo de fora indireta.

Skinner Phillips ${ }^{1}$, referindo-se ao assunto dizem: «o aquecimento do revestimento à $700^{\circ} \mathrm{C}$ não faz com que sua resistência seja muito menor do que aquela apresentada à temperatura ambiente. Após ter es-

- Professor Catedrático de Materiais Dentários, Faculdade de Odontologia, UFRGS

* Professor Auxiliar de Ensino de Materiais Dentários, Faculdade de Odontologia, UFRGN, Bolsa de Aperfeiçoamento de Ensino e Pesquisa concedida pela CAPES. 
friado à temperatura ambiente, aresistência diminui consideravelmente, talvez devido às pequenas fraturas que se fomam durante o esfriamento.

Souder e Paffenbarger 2, efetuaram ensaios de resistência à compressão de dois tipos de revestimento durante a elevação de temperatura e após o seu total resfriamento, chegando à conclusão de que existe uma diminuição bastante significativa na resistência à compressão.

Outra referência que se relaciona com o presente trabalho, ainda que indiretamente, é o gráfico (p. 166) bem como a tabela (p. 167) encontrados no livro de Smith $^{3}$, nos quais o referido autor relaciona as condições de temperatura de um revestimento com sua resistência à compressão, sem contudo dar maiores detalhes sobre a forma como foi conduzida a investigação.

O objetivo da presente investigaçăo é o de estudar o comportamento de quatro marcas de revestimento no que se relaciona à sua resistência ao impacto, quándo retirados do forno à temperatura de $700^{\circ} \mathrm{C}$.

0 tempo decorrido deste à retirada do forno e o momento em que se realizavam os ensaios de resistência ao impacto, era respectivamente de: $15,30,40,50$ e 60 segundos e, 5, 10 e 15 minutos.

\section{MATERIAIS E MÉTODOS}

Os corpos de prova eram obtidos, vasando-se as misturas de revestimento, em sua consistência padrão, de acordo com as normas geralmente aceitas para este fim. 4 e $^{5}$

As quatro marcas de revestimento utilizados nesta pesquisa, bem como as proporções água/revestimento em que foram empregados e os nomes de seus respectivos fabricantes, constam da Tabela de No 1 .

$\begin{array}{llllllllll} & \mathbf{A} & \mathbf{B} & \mathbf{E} & \mathbf{L} & \mathbf{A} & \mathbf{N}^{\rho} & \mathbf{1}\end{array}$

\begin{tabular}{l|c|c|l|c}
\hline M A R C A & SIGLA & Proporção A/P & FABRICANTE & $\begin{array}{c}\text { Parti- } \\
\text { da (No) }\end{array}$ \\
\hline HERODENT & A & 0,33 & Herman Josias S.A. & $\mathrm{n} / \mathrm{c}$ \\
\hline EXCELSIOR & $\mathrm{B}$ & 0,35 & S.S. White Company & $\mathrm{n} / \mathrm{c}$ \\
\hline $\begin{array}{l}\text { EXPERIMENTAL } \\
\text { S. S. WHITE }\end{array}$ & $\mathrm{C}$ & 0,40 & S.S. White Company & $\mathrm{n} / \mathrm{c}$ \\
\hline \begin{tabular}{l} 
CRISTOBALITE \\
\hline
\end{tabular} & $\mathrm{D}$ & 0,40 & Kerr Manuf. Co. & $\mathrm{n} / \mathrm{c}$ \\
\hline
\end{tabular}

R. Fac. Odont. P. A. 


\section{Z 1 ELABORAÇ̃̃O DOS CORPOS DE PROVA}

Os corpos de prova foram elaburados numa matriz de ação inoxidáveı, segmentada, que possuía as seguintes dimensões: $70 \mathrm{~mm}$ de comprimento, $12 \mathrm{~mm}$ de altura e $12 \mathrm{~mm}$ de largura. O volume era consequentemente de $10.080 \mathrm{~mm}^{3}$.

A matriz de aço inoxidável era presa por um atarrachador em forma «C», e depositada sobre uma lage de vidro. Uma vez vasada a mistura de revestimento, em sua consistência padrão (conforme Tab. 1), colocavamos uma outra lage de vidro sobre a matriz, pressionada por um peso de $1.680 \mathrm{~g}$, afim de que os corpos de prova tivessem superfícies planas e paralelas.
Nos ensaios de perda de temperatura, inseria-se no centro geométrico do corpo de prova, um terminal de par termo-elétrico.

Decorridos 15 minutos, retirava-se o atarrachador, separando-se a matriz de aço inoxidável, liberando assim o corpo de prova.

$\mathrm{Na}$ figura de $\mathrm{N}^{\circ} 1$, podemos observar a matriz utilizada, bem como 3 corpos de prova, já elaborados.

\subsection{ENSAIOS DE PERDA TEMPERATURA}

Antes de realizarmos os ensaios de resistência ao impacto, realizamos uma série de ensaios para verificar a perda de temperatura nos revestimentos, à partir de $700^{\circ} \mathrm{C}$.

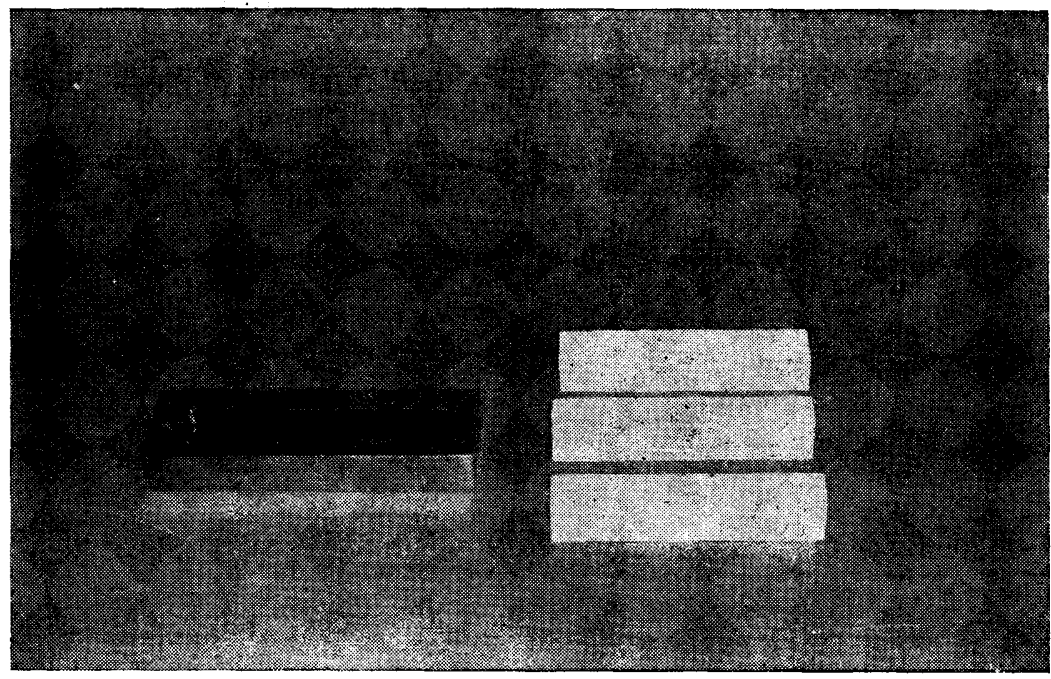

Fig. 1 - Matriz de aço inoxidável, utilizada na elaboração dos corpos de prova.

R. Fac. Odont. P. A.

$13 / 14: 87-97,1971 / 1972$ 
Determinamos a perda de tempera. tura, através de um par termo-elétrico «Cromel-Alumel»-Miliamperimetro, com o terminal colocado no centro geométrico do corpo de prova, loca lem que o mesmo seria submetido à cargas de impacto. (Veja figuras 2 e 3)."

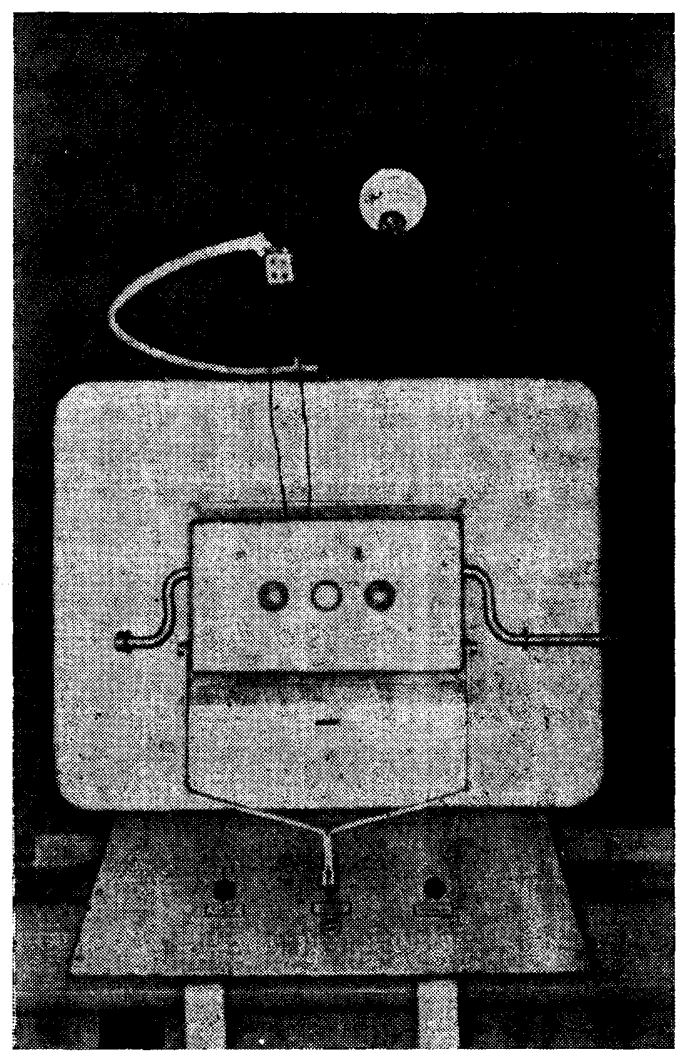

Fig. 2 - Forno e Par termo-elétrico, utilizados na verificação da perda de temperatura dos Corpos de Prova. 


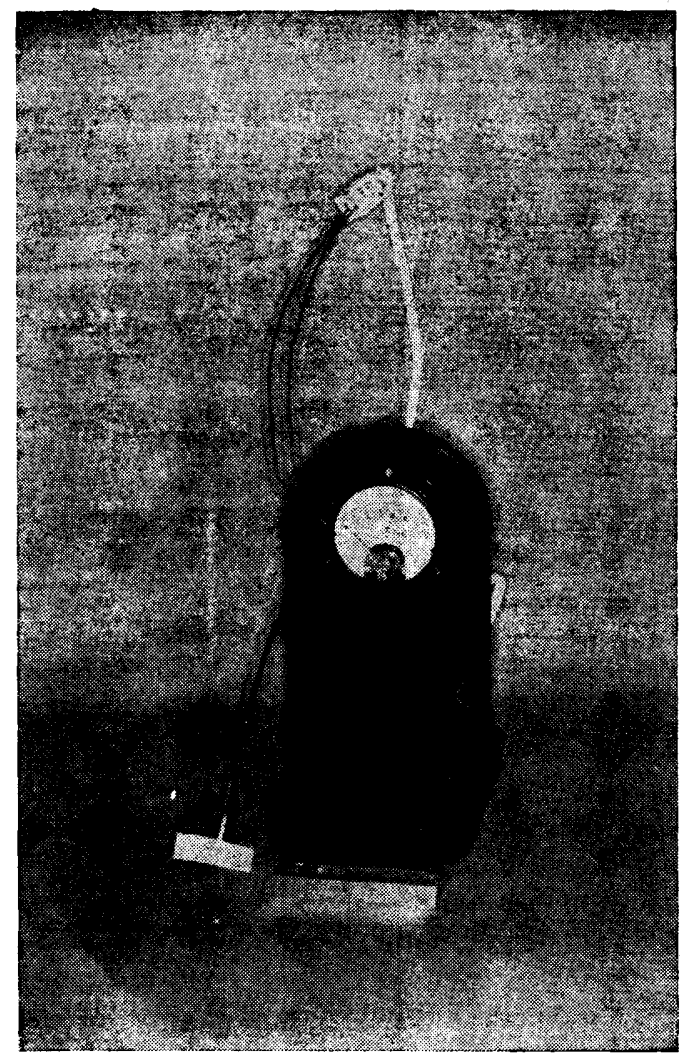

Fig. 3 - Par termo-elétrico com sua terminal colocada no centro geométrico do Corpo de Prova, lo. cal em que o mesmo seria submetido à cargas de impacto.

As leituras da quantidade de calor perdida, eram efetuadas nos diversos espaços de tempo decorridos após a retirada dos corpos de prova do forno, conforme consta da Tabela de $\mathrm{N}^{\circ} 2$.

R. Fac. Odont. P. A. 


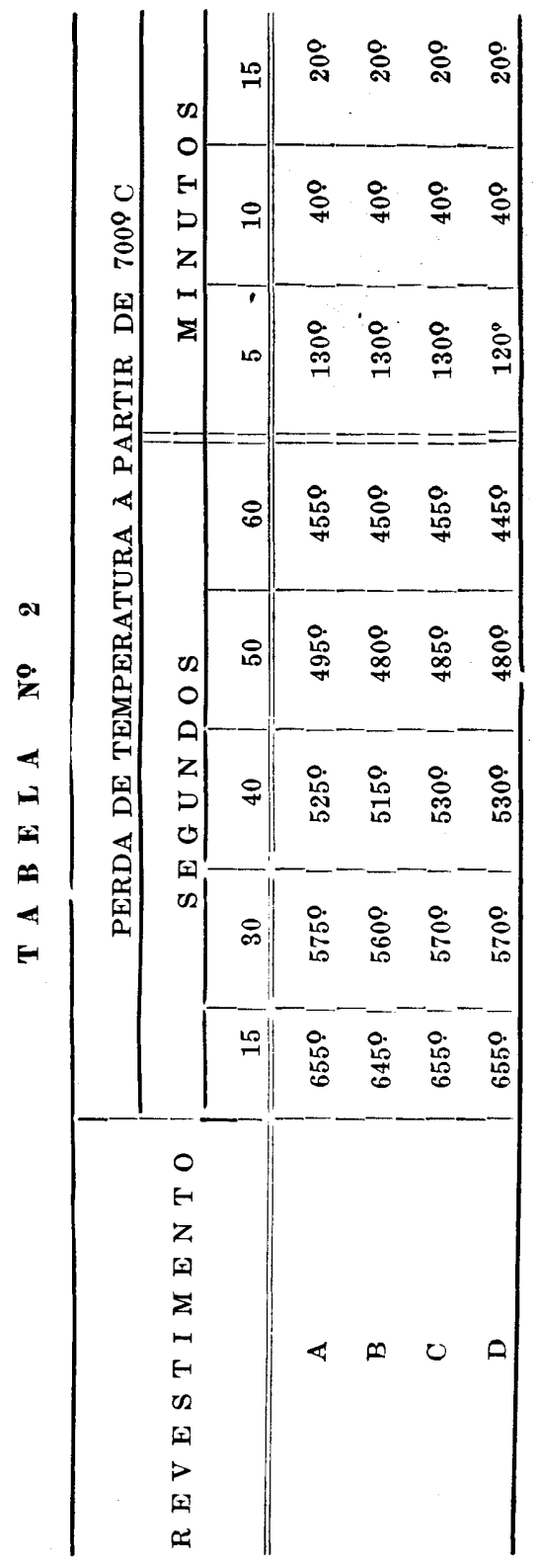

\subsection{ENSAIOS DE RESISTENCIA AO IMPACTO}

Cada corpo de prova era colocado no forno, à temperatura ambiente, aguardando-se que o mesmo atingisse a temperatura de $700^{\circ} \mathrm{C}$ (Fig. 4)

Decorridos 10 minutos nesta temperatura, o corpo de prova era retirado do forno e aguardávamos espaços de tempo de respectivamente: $15,30,40,50$ e 60 segundos e 5,10 e 15 minutos, para procedermos ao ensaio de resistência ao impacto, em aparelhagem adequada. (Fig. 5)

A potência de trabalho da máquina de resistência ao impacto que usamos era de $0,05 \mathrm{Kpm}$. Uma vez rompido o corpo de prova, anotava-se a carga de ruptura e, para obtermos o valor da resistência ao impacto do corpo de prova, aplicavamos a seguinte fórmula:

$$
\begin{aligned}
& \text { A } \\
& \mathrm{a}_{\mathrm{n}}=\frac{\mathrm{n}}{\mathrm{b} \times \mathrm{h}} \text { em que: } \\
& \text { a }=\text { resistincia ao impacto } \\
& \text { n } \quad\left(\mathrm{Kp} / \mathrm{cm}^{2}\right) \\
& A_{\mathrm{n}}=\underset{(\mathrm{Kp} / \mathrm{cm})}{\operatorname{trabalho}} \text { de impacto realizado } \\
& \text { b = largura do corpo de prova em } \\
& \mathrm{cm} \text { no seu centro } \\
& \mathrm{h}=\text { altura do corpo de prova em }
\end{aligned}
$$




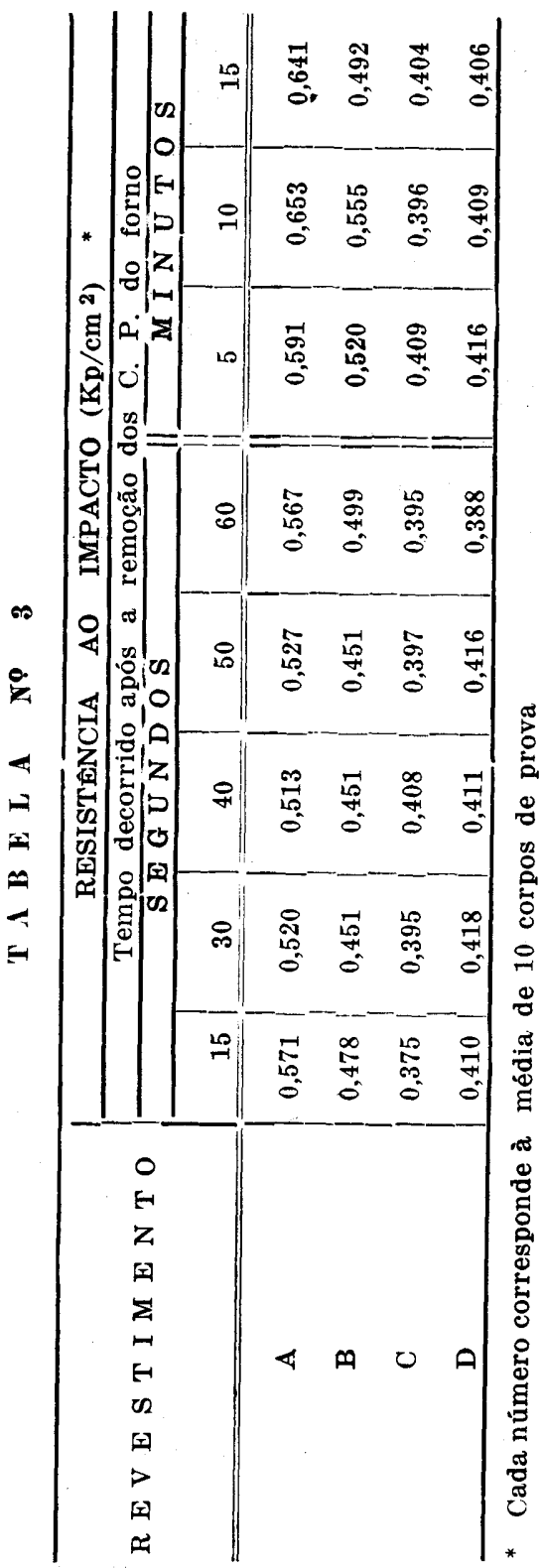

\section{RESULTADOS OBTIDOS E IN. TERPRETACAO DOS MESMOS}

Os resultados obtidos podem ser observados na Tabela de $\mathrm{N}^{\circ} 3$ bem como no Gráfico de $\mathrm{N}^{\mathbf{9}}$ I

\section{1 - A representação gráfica da} Tabels de No 3 (Gráfico I) examinada a primeira vista, nos permitiria observar em primeiro lugar que as resistências ao impacto são superiores no revestimento $\mathrm{HE}$ RODENT, seguindo-se em ordem o revestimento EXCELSIOR, EXPERIMENTAL S. S. WHITE e CRISTOBALITE.

Em segundo lugar, chama-nos atenção o fato de que as tendências das curvas dos revestimentos que contém quartzo em sua composição (HERODENT e EXCELSIOR) são iguais, ou seja, inicialmente há um decréscimo, seguido de um aumento à partir de 50 segundos, para decrescer novamente à patir do $10^{\circ} \mathrm{mi}-$ nuto. Por outro lado e, com resistências ao impacto menores, encontramos, bastante similares, as curvas dos revestimentos que contém a silica em sua variedade alotrópica cristobalita. (EXPERIMENTAL S. S. WHITE e CRISTOBALITE) Salientamos o fato de que cada ponto de cada uma das curvas no gráfico I, representa média de 10 ensaios.

3.1 - Depois de elaborar o gráfico I, submetemos todos os resultados à análise estatística (análise de variância - distribuição de «F» e diferença mínima significativa). 


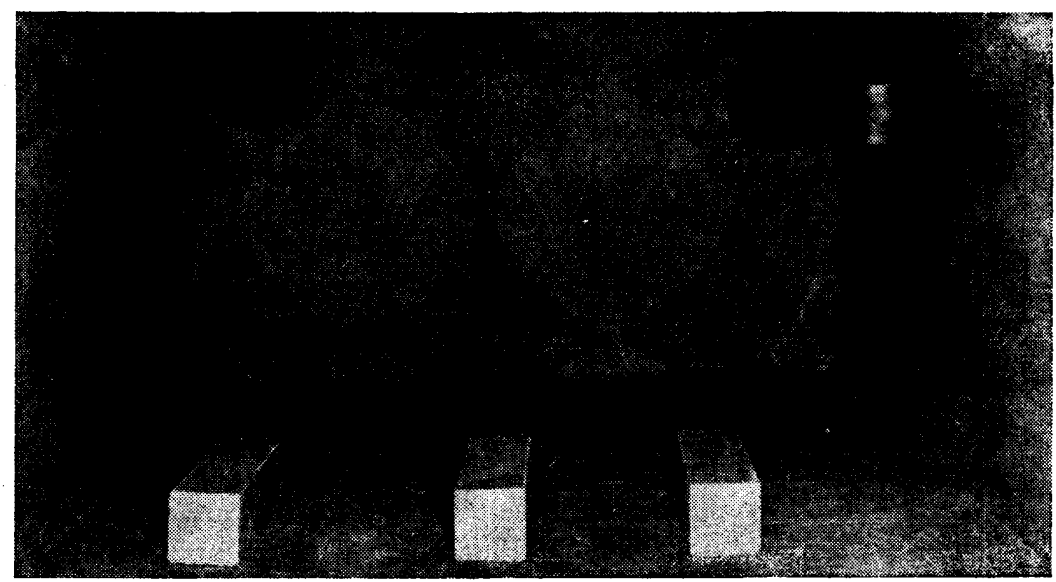

Fig. 4 - Três Corpos de Prova no interior do forno.

Verificamos, então, como é fácil nos iludirmos pela interpretação de curvas elaboradas simplesmente à partir de médias de ensaios!

o que, no entanto, a análise estatística nos revelou, considerando cada um dos revestimentos separadamente, bem como comparando os quatro revestimento, à cada um dos espaços de tempo decorridos após a remoção do forno à $700^{\circ} \mathrm{C}$, permitiunos as conclusões adiante relacionadas.

\section{CONCLUSOES}

\subsection{HERODENT}

1 - A diminuição na resistência ao impacto entre o momento da remoção do forno, até aos 40 segundos é estatisticamente significativa ao nível de .05 .
2 - 0 aumento na resistência ao impacto à partir dos 40 segundos e os 5,10 e 15 minutos posteriores, é estatisticamente significativo ao nivel de .01.

3 - Verifica-se o aumento na resistência ao impacto entre 60 segundos e 10 minutos, aumento este significativo ao nível de .01 .

4 - Entre os 60 segundos e os 15 minutos, o aumento também é significativo ao nível de .01 .

\section{EXCELSIOR}

1 - Não há diferenças significativas na resistência ao impacto, desde a sua remoção do forno, até aos 60 segundos.

2 - Verifica-se um aumento significativo, ao nivel de .01 entre os 60 segundos e os 10 minutos.

R. Fac. Odont. P. A. 


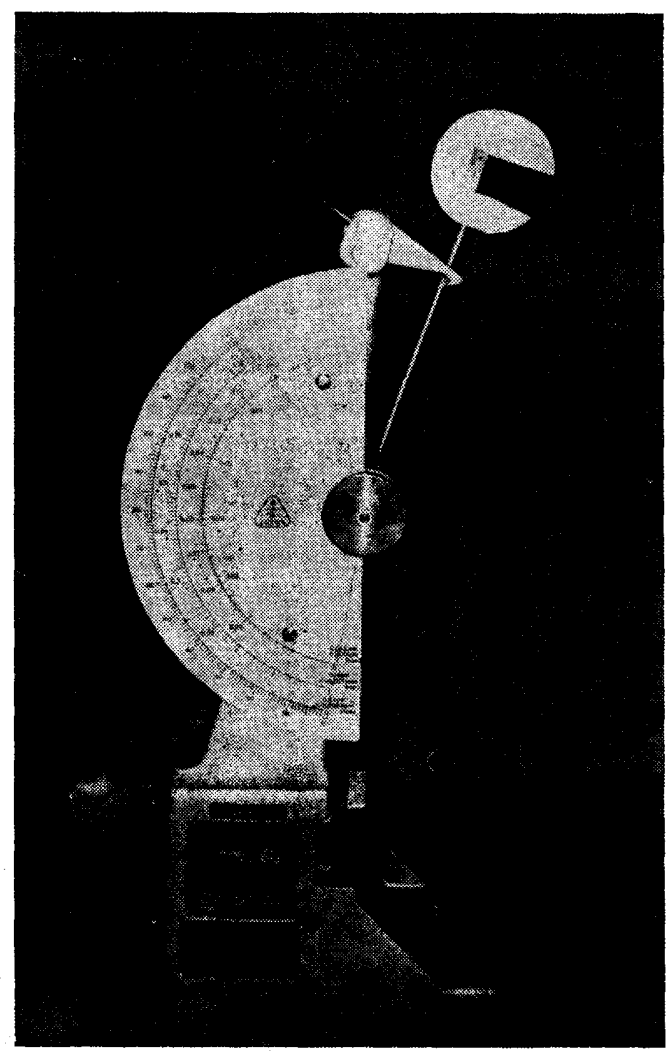

Fig. 5 - Aparelhagem, com o Corpo de Prova em posição para a verificação da resistência ao impacto.

3 - Entre 30, 40 e 50 segundos, comparados com 5 e 10 minutos após a remoção do forno, há uma diferença significativa ao nível de .01.

EXPERIMENTAL S. S. WHITE

1 - Não há diferença estatistica- 1 - Não há diferença estatistica- mente significativa entre os valores de resistência ao impacto deste revestimento, desde a sua remoção do forno, até decorridos 15 minutos.

\section{CRISTOBALITE}

$13 / 14: 87-97,1971 / 1972$

R. Fac. Odont. P. A. 


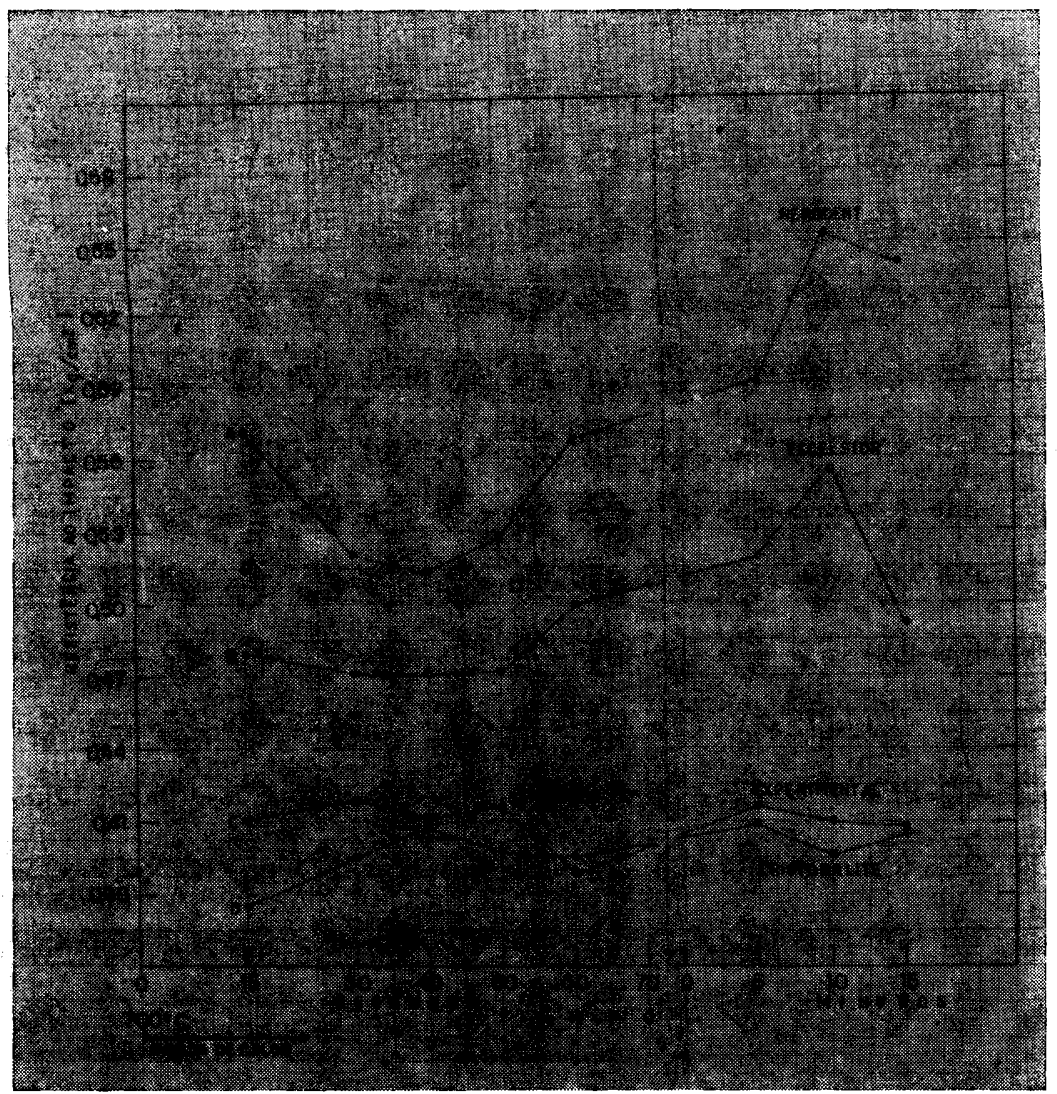

GRAFICO I - Resistência ao impacto de Revestimentos em função da perda de calor a partir de $7009 \mathrm{C}$

mente significativa entre os valores de resistência ao impacto deste revestimento, desde a sua remoção do forno, até decorridos 15 minutos.

4.2 Comparando os quatro revestimentos, a cada um dos espaços detempo decorridos após a remoção do forno, a análise estatística nos revelou o seguinte:
1 - Em nenhum dos espaços de tempo decorridos após a remoção do forno, houve diferença significativa entre as resistências ao impacto dos revestimentos EXPERIMENTAL S. S. WHITE $\Theta$ CRISTOBALITE.

2 - Houve diferenca significativa ao nivel de .05 entre os revestimentos EXCELSIOR e EXPE-

R. Fac. Odont. P. A.

$13 / 14: 87-97,1971 / 1972$ 
RIMENTAL S. S. WHITE (15 e 30 segundos) e HERODENT e EXCELSIOR (5 minutos).

3 - Em todos os demáis casos, considerando-se os espaços de tempo decorridos após a remoção do forno, houve diferenças significativas ao nível de .01, quanto às resistências ao impacto, entre os revestimentos HERODENT A EXCELSIOR, HERODENT A EXPERIMENTAL S. S. WHITE, EXCELSIOR e CRISTOBALITE.

\section{RESUMO}

0 comportamento de quatro marcas de revestimento odontológico, no que se relaciona à sua resistência ao impacto, quando retirados do forno à temperatura de $700^{\circ} \mathrm{C}$ e, decorridos espaços de tempo variáveis desde 15 , $30,40,50$ e 60 segundos e, 5, 10 e 15 minutos, foi estudado. 0 relacionamento gráfico do total das experiências, na forma de curvas elabo- radas simplesmente à partir de médias, ainda que de 10 ensaios cada uma, quando não seguido de uma análise estatística, facilmente permitirá deduções e conclusões errôneas. Ficou demonstrado que o revestimento EXPERIMENTAL S. S. WHITE, de fabricação nacional em fase experimental, se comporta, em relação à sua resistência a impacto, de maneira em tudo idêntica à seu similar estrangeiro, denominado Cristobalite.

\section{S Y N O P S I S}

The impact strenght of four dental casting investments is investigated as a function of heat loss after removal of a furnace temperature of $7^{\circ} 0^{\circ} \mathrm{C}$, at different time intervals. Attention is called to the fact hat $a$ graphical relationship between temperature loss and impac strenght, even hough obtained from averages of 10 experiments, but without a statistical analysis, may induce to erroneous conclusions.

\section{REFERENCIAS BIBLIOGRAFICAS}

1. SKINNER, E.W. \& PHILLIPS, R.W. - La ciencia de los materiales dentales. 6.ed. Buenos Aires, Mundi, 1970. 639p.

2. SOUDER, W. \& PAFFENBARGER, G.C. - Physical properties of dental materials. Washington, National Bureau of Standards, 1942, p.88 (Circular C433)

3. SMITH, Cafpbell J. - The chemistry and metallurgy of dental materials. 2.ed. London, Blackwell Scientific Pub., 1949, p.88

4. AMERICAN DENTAL ASSOCIATION, COUNCIL ON DENTAL MATERIALS AND DEVICES. - Guide to dental materials and devices, 1968-69. 4.ed. Chicago, 1968, p.125-29.

5. GRUPO BRASILEIRO DE MATERIAIS DENTARIOS - Especificações brasileiras, no 11: especificações para revestimentos para fundições de liga de ouro odontológicas. Porto Alegre, 1966, p.1-5. 